\section{Characteristics of aniline and nitrobenzene adsorption on single-walled, multi-walled and graphitized multi-walled carbon nanotubes}

\author{
Shuai Liu, Yifei Zha, Yuanyuan Wang, Qi Wei, \\ Ying Zhang, Yuankai Zhang*, Hongchen Wang, \\ Lu Qi and Xianglong $X u$
}

School of Environment and Natural Resources, Renmin University of China, No. 59 Zhongguancun Street, Beijing 100872, China

Herein, we assess the relative potential of singlewalled, multi-walled and graphitized multi-walled carbon nanotubes (CNTs) for the adsorptive removal of organic species using aniline and nitrobenzene as model aromatic contaminants. The obtained results reveal that the adsorption behaviour of aromatic organics is significantly affected by their functional groups (especially when functionalized CNTs are used as adsorbent), providing valuable guidance for adsorption-based removal of organic contaminants from the environment.

Keywords: Adsorption, aromatic organics, carbon nanotubes, relative potential.

POLLUTION caused by the presence of aromatic organics in the natural environment is a global problem that requires urgent attention and currently presents a technological challenge. The non-biodegradability of the above species causes their accumulation in the environment and can lead to severe contamination of soil and water $^{1,2}$, posing a serious ecological threat and necessitating the development of efficient removal technologies, e.g. those based on adsorption ${ }^{3}$, advanced oxidation ${ }^{4}$, membrane separation ${ }^{5}$ and bioremediation ${ }^{6}$. Some carbonaceous materials made from plant residues $\left(\right.$ cone $^{7}$, peel $^{8}$ and shell $^{9}$ ) showed excellent biosorption of chromium (VI), and a dried fungal biomass performed well on the removal of turquoise blue dye ${ }^{10}$. Among these techniques, adsorption is considered to be superior to other decontamination methods because of its simplicity, high efficiency and ease of operation.

Although activated carbon has an excellent adsorption capacity for organic pollutants and is, therefore, the most commonly used commercial adsorbent ${ }^{11,12}$, it exhibits certain disadvantages, including low adsorption capacity and difficulty of recovery. Recently, new carbonaceous nanomaterials have attracted increased attention as potential adsorbents for water treatment, and are considered to be a viable alternative to traditional adsorbents such as activated carbon. Among these nanomaterials, carbon

\footnotetext{
*For correspondence. (e-mail: zyk11@ruc.edu.cn)
}

nanotubes (CNTs, discovered by Iijima ${ }^{13}$ in 1991) are one of the most widely studied adsorbents ${ }^{14-16}$, featuring the advantages of a hollow and layered structure, high specific surface area and superior hydrophobicity, and thus showing strong adsorption affinities to a wide range of organic contaminants ${ }^{17-20}$. Moreover, the large external surface area and well-developed mesopores of CNTs make them superior and particularly promising adsorbents for the removal of microorganisms, natural organic matter and toxins from drinking water ${ }^{21-24}$.

Depending on their structure/functionalization, CNTs can be classified into single-walled CNTs (SWCNTs), multi-walled CNTs (MWCNTs) and functionalized $\mathrm{CNTs}^{25-28}$, with the first two classes being those most widely used ${ }^{13,29}$. CNTs can be visualized as graphitic carbon sheets rolled into hollow cylinders with nanometre-scale diameters and micrometre-scale lengths ${ }^{30}$. SWCNTs possess a single-layer structure and higher uniformity, whereas MWCNTs features a multilayer structure, greater structural stability and a higher pore packing effect, which results in physical property differences between these CNTs ${ }^{31}$. The adsorption of aromatic compounds by CNTs is strongly influenced by the chemical properties of the CNT surface, corresponding to both chemical and physical (mainly van der Waals forcedriven) adsorption, and involving hydrogen bonding and $\pi-\pi$ electron donor-acceptor (EDA) interactions ${ }^{32-34}$. Graphitized multi-walled CNTs (GMWCNTs) have a much smaller content of oxygenated surface functional groups compared to MWCNTs ${ }^{35}$ and thus exhibit different adsorption behaviour and strong hydrophobicity. Therefore, to further study the factors affecting the adsorption behaviour of CNTs, we herein use singlewalled, multi-walled and graphitized multi-walled carbon nanotubes for adsorption experiments.

In this study, two typical aromatic compounds with different functional groups, namely aniline and nitrobenzene, were used to elucidate the factors controlling the adsorption behaviour of CNTs, and the substituents of these organics were shown to influence their adsorption. Specifically, the fact that aniline is ionizable, whereas nitrobenzene is not, implies that the solution-phase adsorption of the former on CNTs involves both physical and chemical adsorption. In addition, the strongly electron-withdrawing nature of the nitro group was shown to reduce the $\pi$-electron density of the benzene ring and affect the $\pi-\pi$ EDA interaction. The obtained results shed light on the factors influencing the adsorption process, and can be used as a reference to determine appropriate adsorbents for the abatement of pollution caused by aromatic organics.

SWCNTs, MWCNTs and GMWCNTs (all >99.0\%) were purchased from Chengdu Organic Chemicals Co. Ltd, China and were used as received. Prior to experiments, CNTs were dispersed in Milli-Q water and sonicated for $15 \mathrm{~min}$. Brunauer-Emmett-Teller (BET) analysis 
Table 1. Selected properties of the studied carbon nanotubes (CNTs)

\begin{tabular}{|c|c|c|c|c|c|c|}
\hline \multirow[b]{2}{*}{ CNTs } & \multirow[b]{2}{*}{$V_{\mathrm{TP}}\left(\mathrm{cm}^{3} \mathrm{~g}^{-1}\right)^{\mathrm{a}}$} & \multirow[b]{2}{*}{$D_{\mathrm{mp}}(\mathrm{nm})^{\mathrm{b}}$} & \multicolumn{3}{|c|}{$\begin{array}{l}\text { Content of surface oxygen } \\
\text { functional groups }\left(\mathrm{m} \mathrm{mol} \mathrm{g}^{-1}\right)\end{array}$} & \multirow[b]{2}{*}{$\mathrm{pH}_{\mathrm{pzc}}{ }^{\mathrm{c}}$} \\
\hline & & & Hydroxyl & Carboxyl & Total & \\
\hline Single-walled CNTs & 0.29 & 5.45 & 0.025 & 0.038 & 0.063 & 3.9 \\
\hline Multi-walled CNTs & 0.23 & 6.47 & 0.013 & 0.134 & 0.147 & 5.3 \\
\hline Graphitized multi-walled CNTs & 0.13 & 5.38 & 0.003 & 0.005 & 0.008 & 6.5 \\
\hline
\end{tabular}

${ }^{\mathrm{a}}$ Total pore volume calculated from single-point adsorption for $P / P_{0}=0.99$. ${ }^{\mathrm{b}}$ Mean pore diameter obtained using the BET model. ${ }^{c}$ Point of zero charge (pzc) obtained by batch adsorption tests.

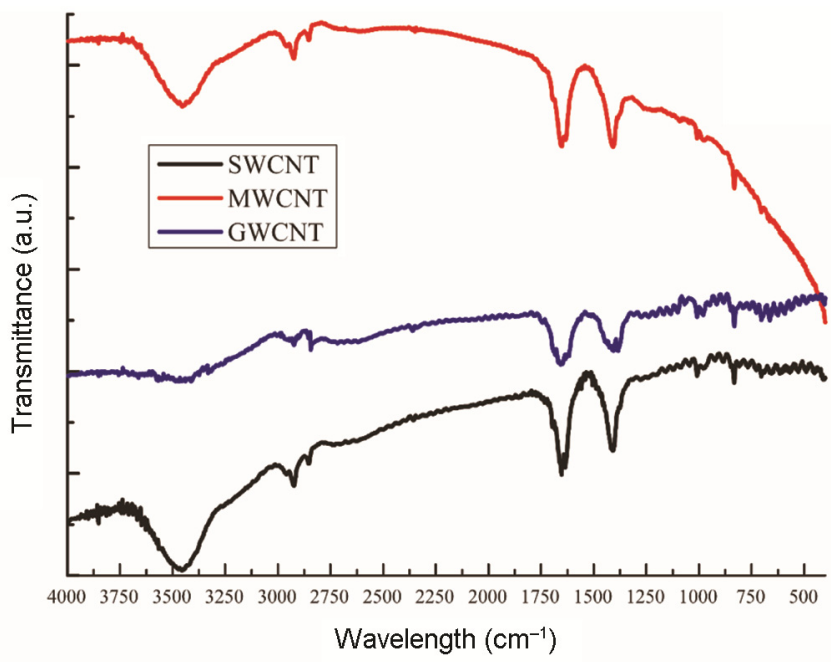

Figure 1. Fourier transform infrared spectra of single-walled carbon nanotubes (SWCNTs), multi-walled carbon nanotubes (MWCNTs) and Graphitized multi-walled carbon nanotubes (GMWCNTs).

(Tristar 3020 II, micromeritics) and Fourier transform infrared (FTIR) spectroscopy (Bruker TENSOR 27) were used to study the surface properties of the three CNT types. In addition, the CNTs were characterized by zeta potential $\left(\mathrm{pH}_{\mathrm{pzc}}\right)$ measurements.

Adsorption experiments were performed using single aromatic compounds (aniline or nitrobenzene) to prevent competitive adsorption. Stock solutions $\left(200 \mathrm{mg} \mathrm{l}^{-1}\right)$ of aniline and nitrobenzene (Sinopharm Chemical Reagent Co. Ltd) were separately prepared in ultra-pure water and stored at room temperature. $\mathrm{NaCl}, \mathrm{NaOH}, \mathrm{HCl}, \mathrm{KBr}$ and other analytical-grade reagents were purchased from Sinopharm Chemical Reagent Co. Ltd, China. The solution $\mathrm{pH}$ in all tests was adjusted using $1 \mathrm{M} \mathrm{HCl}$ and $1 \mathrm{M} \mathrm{NaOH}$.

Experiments for studying adsorption kinetics were performed at a constant CNT concentration of $200 \mathrm{mg} \mathrm{l}^{-1}$ and a constant temperature of $25^{\circ} \mathrm{C}$ in a water bath at a stirring rate of $250 \mathrm{rpm}$. For isotherm studies, temperatures of $15^{\circ} \mathrm{C}, 25^{\circ} \mathrm{C}$ and $35^{\circ} \mathrm{C}$ were used, and the effects of $\mathrm{pH}$ were probed using $\mathrm{pH}$ values of $2,3,5,7,9,11$ and 13, with other parameters being constant.

All mixtures were stirred for a maximum of $6 \mathrm{~h}$, centrifuged for $5 \mathrm{~min}$ at $14,000 \mathrm{rpm}$ and passed through a
$0.45 \mu \mathrm{m}$ filter. The concentration of aniline or nitrobenzene in the filtrate was measured using a fluorescence spectrophotometer. Each experiment was performed in duplicate, and the average was calculated. Adsorption was described by the Freundlich model.

The BET surface areas $\left(\mathrm{SA}_{\mathrm{BET}}\right)$ and pore size distributions of the studied CNTs were estimated based on $\mathrm{N}_{2}$ adsorption/desorption isotherms recorded at $77 \mathrm{~K}$ (Tristar 3020 II, Micromeritics) using built-in software. Total pore volume and mean pore diameter were estimated using the Barrett-Joiner-Halenda equation during the desorption phase ${ }^{36}$. SWCNTs, MWCNTs and GMWCNTs exhibited surface area of 210.5, 142.3 and $97.7 \mathrm{~m}^{2} \mathrm{~g}^{-1}$ respectively, total pore volume of $0.29,0.23$ and $0.13 \mathrm{~cm}^{3} \mathrm{~g}^{-1}$ respectively, and mean pore diameter of $5.45,6.47$ and $5.38 \mathrm{~nm}$ respectively (Table 1).

CNTs contain a number of surface functional groups (e.g. $-\mathrm{OH},-\mathrm{COOH}$ and $-\mathrm{C}=\mathrm{O}$ ) that might engage in interactions with adsorbates. Herein, CNTs were analysed by FTIR spectroscopy to confirm the possible effects of functional groups on adsorption properties. Specifically, the $\mathrm{KBr}$ pressed-disk technique was used and spectra were recorded for a wavenumber range $400-4000 \mathrm{~cm}^{-1}$ at a resolution of $4 \mathrm{~cm}^{-1}$ in transmission mode at room temperature.

The FTIR spectra of the three studied CNTs presented in Figure 1 demonstrate the presence of several surface functional groups. Thus, absorbance bands at $\sim 3450 \mathrm{~cm}^{-1}$ were ascribed to hydroxyl groups, and those at 1650 $1750 \mathrm{~cm}^{-1}$ indicated the presence of carboxyl groups. Both the functional groups were least abundant in the case of GMWCNTs. Bands at $\sim 1450 \mathrm{~cm}^{-1}$ were attributed to $\mathrm{C}-\mathrm{O}$ moieties, and those at $\sim 1000 \mathrm{~cm}^{-1}$ reflected the presence of $\mathrm{C}-\mathrm{C}$ and $\mathrm{C}-\mathrm{H}$ functionalities. Table 1 lists the contents of hydroxyl and carboxyl groups determined by FTIR spectroscopy as well as other physical properties of the studied CNTs. Compared to MWCNTs, GMWCNTs exhibited a smaller surface area, mean pore diameter, total pore volume and content of surface functional groups, which resulted in enhanced hydrophobicity.

The point of zero charge (pzc) of CNTs was obtained using batch adsorption tests. For each CNT type, $5 \mathrm{mg}$ of adsorbent was shaken in polypropylene tubes with $25 \mathrm{ml}$ of $0.02 \mathrm{M} \mathrm{NaCl}$ at background $\mathrm{pH}\left(\mathrm{pH}_{\mathrm{i}}\right)$ values of $2-11$, 

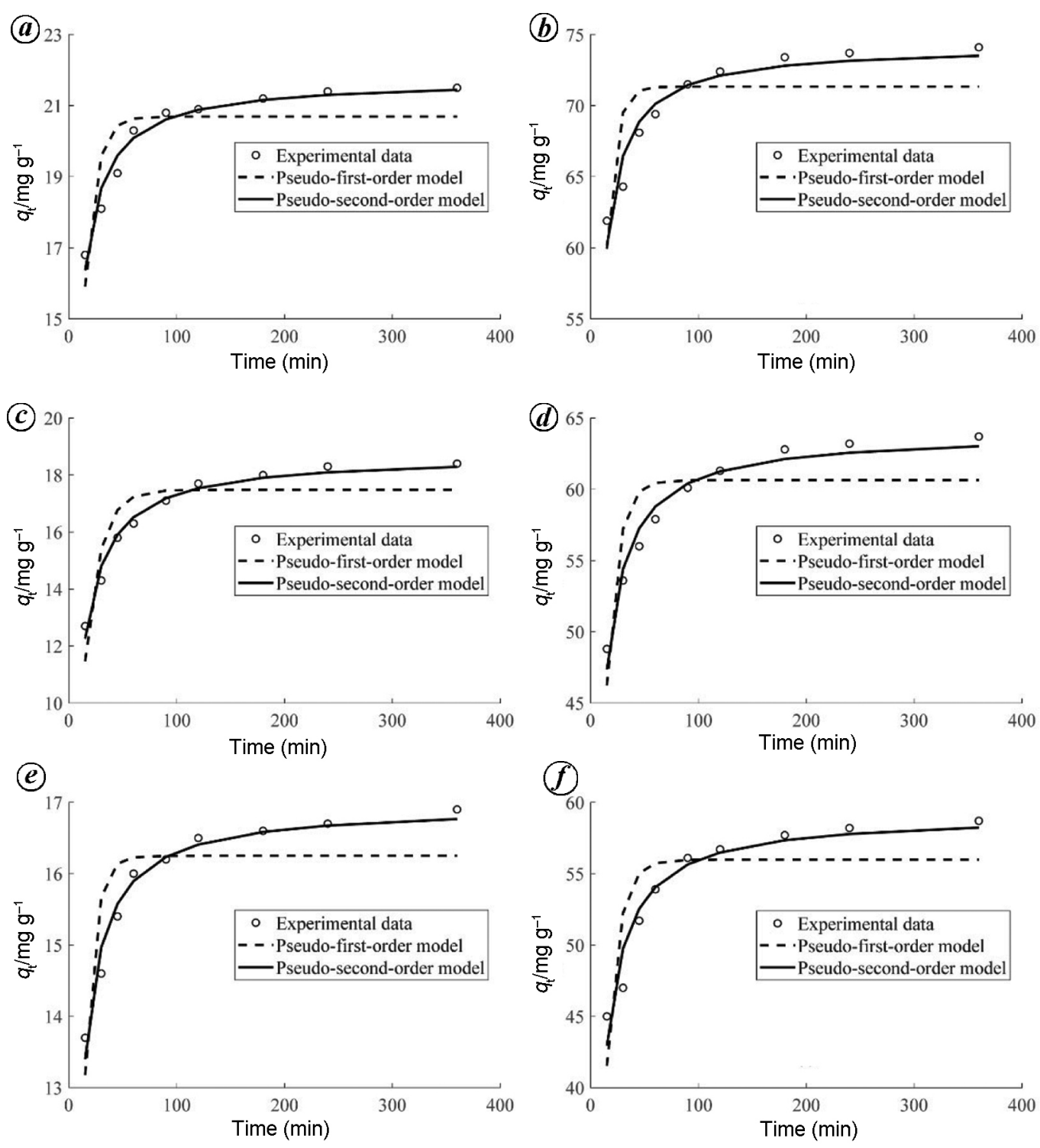

Figure 2. $\boldsymbol{a}-\boldsymbol{c}$, Kinetics of aniline adsorption on (a) SWCNTs, (b) MWCNTs and (c) GMWCNTs. $\boldsymbol{d}-\boldsymbol{f}$ Kinetics of nitrobenzene adsorption on $(\boldsymbol{d})$ SWCNTs, $(\boldsymbol{e})$ MWCNTs and $(\boldsymbol{f})$ GMWCNTs.

and the final $\mathrm{pH}\left(\mathrm{pH}_{\mathrm{f}}\right)$ was measured after $6 \mathrm{~h}$ of shaking at $25^{\circ} \mathrm{C}$. Subsequently, $\Delta \mathrm{pH}\left(\mathrm{pH}_{\mathrm{i}}-\mathrm{pH}_{\mathrm{f}}\right)$ was plotted against $\mathrm{pH}$, and pzc was identified as the intercept of the obtained graph with the $x$-axis. The zeta potential $\left(\mathrm{pH}_{\mathrm{pzc}}\right)$ of SWCNTs, MWCNTs and GMWCNTs (determined as $3.9,5.3$ and 6.5 respectively) indicated that all CNTs were negatively charged at neutral $\mathrm{pH}$, additionally showing that $\mathrm{pH}_{\mathrm{pzc}}$ decreased, and the amount of negative charge increased with increasing content of oxygenated surface functional groups.

A series of experiments were conducted to study the kinetics, isotherms and thermodynamics of aniline and nitrobenzene adsorption by CNTs, and determine the effects of $\mathrm{pH}$. The corresponding adsorption mechanisms were elucidated by fitting experimental kinetic data with pseudo first-order and pseudo second-order models.
The adsorption behaviour of aniline and nitrobenzene was fairly similar for all CNTs. In particular, adsorption capacity increased with time, and adsorption equilibria were reached after $\sim 6 \mathrm{~h}$. Figure 2 indicates an instantaneous adsorption in time initially, gradually leading to equilibrium, similar to previous studies ${ }^{37,38}$. At first, aniline/nitrobenzene was adsorbed or instantaneously adsorbed on the surface of CNTs. As the concentration of aniline/nitrobenzene decreased gradually, the effect of internal diffusion became weaker and adsorption equilibrium was reached. For aniline, the observed equilibrium adsorption capacities were in the order of SWCNTs $\left(21.5 \mathrm{mg} \mathrm{g}^{-1}\right)>$ MWCNTs $\quad\left(18.4 \mathrm{mg} \mathrm{g}^{-1}\right)>$ GMWCNTs $\left(16.9 \mathrm{mg} \mathrm{g}^{-1}\right)$, which agreed with the order of specific surface areas. Conversely, an opposite order was observed for adsorption capacities per unit specific surface 
Table 2. Parameters for pseudo first- and second-order Lagergren models of aniline and nitrobenzene adsorption

\begin{tabular}{|c|c|c|c|c|c|c|c|c|}
\hline \multirow[b]{2}{*}{ Adsorbate } & \multirow[b]{2}{*}{ CNTs } & \multirow[b]{2}{*}{$q_{\mathrm{e}, \exp }\left(\mathrm{mg} \mathrm{g}^{-1}\right)$} & \multicolumn{3}{|c|}{ Pseudo first-order } & \multicolumn{3}{|c|}{ Pseudo second-order } \\
\hline & & & $q_{\mathrm{e}, \mathrm{cal}}\left(\mathrm{mg} \mathrm{g}^{-1}\right)$ & $k_{1}\left(\mathrm{~g} \mathrm{mg} \mathrm{min}^{-1}\right)$ & $R^{2}$ & $q_{\mathrm{e}, \mathrm{cal}}\left(\mathrm{mg} \mathrm{g}^{-1}\right)$ & $k_{2}\left(\mathrm{~g} \mathrm{mg} \mathrm{min}{ }^{-1}\right)$ & $R^{2}$ \\
\hline \multirow[t]{3}{*}{ Aniline } & SWCNTs & 21.5 & 20.7 & 0.0977 & 0.655 & 21.7 & 0.0094 & 0.951 \\
\hline & MWCNTs & 18.4 & 17.5 & 0.0711 & 0.751 & 18.7 & 0.0068 & 0.977 \\
\hline & GMWCNTs & 16.9 & 16.2 & 0.1111 & 0.652 & 17.0 & 0.0149 & 0.964 \\
\hline \multirow[t]{3}{*}{ Nitrobenzene } & SWCNTs & 74.2 & 71.3 & 0.1222 & 0.537 & 74.2 & 0.0039 & 0.927 \\
\hline & MWCNTs & 63.7 & 60.6 & 0.0958 & 0.649 & 63.9 & 0.0030 & 0.963 \\
\hline & GMWCNTs & 58.7 & 56.0 & 0.0903 & 0.598 & 59.1 & 0.0030 & 0.923 \\
\hline
\end{tabular}

area, namely GMWCNTs $\left(0.173 \mathrm{mg} \mathrm{m}^{-2}\right)>$ MWCNTs $\left(0.129 \mathrm{mg} \mathrm{m}^{-2}\right)>$ SWCNTs $\left(0.102 \mathrm{mg} \mathrm{m}^{-2}\right)$. For nitrobenzene, the equilibrium adsorption capacities were in the order of SWCNTs $\left(74.2 \mathrm{mg} \mathrm{g}^{-1}\right)>$ MWCNTs $\left(63.7 \mathrm{mg} \mathrm{g}^{-1}\right)>$ GMWCNTs $\left(58.7 \mathrm{mg} \mathrm{g}^{-1}\right)$, while adsorption capacities per unit specific surface area decreased in the opposite order: GMWCNTs $\left(0.601 \mathrm{mg} \mathrm{m}^{-2}\right)>$ MWCNTs $\left(0.448 \mathrm{mg} \mathrm{m}^{-2}\right)>$ SWCNTs $\left(0.352 \mathrm{mg} \mathrm{m}^{-2}\right)$.

The above results indicated that the adsorption capacity scaled with specific surface area, which implied that hydrophobic interactions (i.e. van der Waals forces) played a major role in the adsorption of aniline on CNTs, in line with previous reports ${ }^{26}$. Additionally, the adsorption capacities per unit specific surface area revealed that the adsorption of aniline and nitrobenzene was also influenced by the nature/content of surface functional groups and other factors.

As shown in Table 2, the kinetics of aniline adsorption on all CNTs was better described by the pseudo secondorder model, which achieved higher correlation coefficients $\left(0.951<R^{2}<0.977\right)$ than the pseudo first-order model $\left(0.652<R^{2}<0.751\right)$. As reported by Mohammadi et al. $^{39}$, the pseudo second-order adsorption rate constants $\left(k_{2}\right)$ were in the order of GMWCNTs $\left(0.0149 \mathrm{~g} \mathrm{mg} \mathrm{min}^{-1}\right)>$

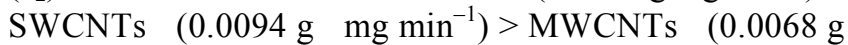
$\left.\mathrm{mg} \mathrm{min}^{-1}\right)$, i.e. the fastest aniline adsorption was observed for GMWCNTs. Since the surface functional group content followed the order of MWCNTs $>$ SWCNTs $>$ GMWCNTs, $k_{2}$ was concluded to be negatively correlated to this parameter, which was explained by the significant influence of hydrogen bonding on adsorption progress. Thus, the presence of oxygenated functional groups was suggested to cause competition between aniline and water for forming hydrogen bonds with surface functional groups and hence decrease the aniline adsorption capacity of CNTs. In the case of strongly hydrophobic GMWCNTs, the formation of hydrogen bonds with water was disfavoured, which increased the number of sites available for aniline adsorption. In summary, the obtained results revealed that the adsorption of aniline on CNTs was influenced by factors such as specific surface area, $\pi-\pi$ aromatic interactions, and hydrogen bonding, and corresponded to a complex chemical adsorption process.
The kinetics of nitrobenzene adsorption on all CNTs was better described by the pseudo second-order model $\left(0.923<R^{2}<0.963\right.$, cf. $0.537<R^{2}<0.649$ for the pseudo first-order model). The pseudo second-order rate constant $\left(k_{2}\right)$ of nitrobenzene adsorption on SWCNTs, MWCNTs and GMWCNTs was determined to be $0.0039,0.0030$ and $0.0030 \mathrm{~g} \mathrm{mg} \mathrm{min}^{-1}$ respectively, i.e. the fastest nitrobenzene adsorption was observed for SWCNTs. Considering the difference of functional group content between MWCNTs and GMWCNTs, the rate of nitrobenzene adsorption was determined to be less affected by the nature/type of surface functional groups than in the case of aniline. Moreover, the fact that SWCNTs exhibited a single-layer structure while MWCNTs and GMWCNTs featured a multi-layer structure, highlighted the significant effect of CNT physical structure on adsorption progress. Since the nitro group is strongly electron-withdrawing, nitrobenzene features a very low electron density in the aromatic ring, whereas the benzene rings of CNTs are $\pi$-electron-rich, which results in $\pi-\pi$ EDA interactions between CNTs and nitrobenzene. In summary, the obtained results showed that the adsorption of nitrobenzene on CNTs was mainly influenced by their physical properties and capability of engaging in $\pi-\pi$ EDA interactions. Consequently, this adsorption was concluded to involve complex heterogeneous diffusion, and be affected by the physical structure of CNTs and chemical bonds formed by functional groups.

Comparably, the nitrobenzene adsorption capacities of the three CNTs were much larger than those observed for aniline and were fairly similar. Previous studies showed that hydrophobic and $\pi-\pi$ aromatic interactions significantly influence adsorption behaviour ${ }^{34,40}$, and demonstrated that organic species with low hydrophobicity do not engage in strong hydrophobic interactions with $\mathrm{CNTs}^{41}$. Thus, the higher nitrobenzene adsorption capacities of CNTs compared to those of aniline were ascribed to the elevated hydrophobicity of the former adsorbate. Moreover, the electron-withdrawing nature of the nitro group in nitrobenzene was thought to enhance $\pi-\pi$ interactions between nitrobenzene and CNTs by decreasing the $\pi$-electron density of the aromatic ring in the former. The similarity of nitrobenzene adsorption capacities 
Table 3. Freundlich model fitting parameters obtained for the adsorption of aniline and nitrobenzene on CNTs

\begin{tabular}{|c|c|c|c|c|c|}
\hline Adsorbate & CNTs & $T\left({ }^{\circ} \mathrm{C}\right)$ & $K_{\mathrm{F}}\left(\mathrm{mg} \mathrm{g}^{-1}\right)$ & $n$ & $R^{2}$ \\
\hline \multirow[t]{9}{*}{ Aniline } & SWCNTs & 25 & 2.16 & 0.777 & 0.982 \\
\hline & & 35 & 0.69 & 1.060 & 0.961 \\
\hline & & 45 & 0.59 & 0.861 & 0.949 \\
\hline & MWCNTs & 25 & 1.19 & 0.896 & 0.990 \\
\hline & & 35 & 0.47 & 1.025 & 0.970 \\
\hline & & 45 & 0.47 & 0.858 & 0.971 \\
\hline & GMWCNTs & 25 & 0.92 & 0.936 & 0.990 \\
\hline & & 35 & 0.41 & 1.006 & 0.980 \\
\hline & & 45 & 0.61 & 0.764 & 0.939 \\
\hline \multirow[t]{9}{*}{ Nitrobenzene } & SWCNTs & 25 & 4.76 & 0.943 & 0.993 \\
\hline & & 35 & 3.22 & 0.940 & 0.965 \\
\hline & & 45 & 3.79 & 0.849 & 0.983 \\
\hline & MWCNTs & 25 & 5.15 & 0.886 & 0.980 \\
\hline & & 35 & 2.98 & 0.938 & 0.989 \\
\hline & & 45 & 3.02 & 0.910 & 0.957 \\
\hline & GMWCNTs & 25 & 2.17 & 1.070 & 0.977 \\
\hline & & 35 & 1.70 & 1.096 & 0.934 \\
\hline & & 45 & 1.06 & 1.192 & 0.965 \\
\hline
\end{tabular}

determined for all CNTs implied that the effect of chemisorption, in this case, was smaller than that for aniline.

The Freundlich and Langmuir model had been applied for the adsorption isotherm of aniline and nitrobenzene on CNTs. However, compared to the Freundlich model, the fitting results of the Langmuir model were rarely poor; the $R^{2}$ for each adsorption was less than 0.9 . Table 3 shows that the Freundlich model is well suited for fitting experiment data $\left(R^{2}=0.934-0.993\right)$, and reveals that the adsorption of aniline and nitrobenzene on CNTs corresponds to multi-layer molecular adsorption. The maximum aniline/nitrobenzene adsorption capacity of the three CNTs gradually decreased with increasing temperature, which indicated that the adsorption process was grossly exothermic. In the Freundlich adsorption isotherm model, the degree of isotherm deviation from linearity is represented by $1 / n$, which is negatively correlated with adsorption performance. At $1 / n<1$, adsorption is relatively easy, whereas the reverse is true for $1 / n>1$. For aniline adsorption, $n$ was mostly less than unity, i.e. the isotherm of aniline adsorption on the three CNTs was highly nonlinear, which was ascribed to the heterogeneous nature of CNT adsorption sites. However, for nitrobenzene adsorption at all studied temperatures, $1 / n$ values of greater than unity were observed for SWCNTs and MWCNTs, whereas these values were less than unity for GMWCNTs, which suggested that the adsorption of nitrobenzene on GMWCNTs was relatively easy, probably because of the increased surface hydrophobicity of these CNTs.

Previously, the presence of oxygenated functional groups on the surface of CNTs was shown to play a significant role in the adsorption of aniline, since carboxyl
$(-\mathrm{COOH})$ and hydroxyl $(-\mathrm{OH})$ moieties are capable of interacting with amino groups $\left(-\mathrm{NH}_{2}\right)$ via hydrogen bonding and Lewis acid-base interactions ${ }^{42}$. Because aniline contains an amino group, and all CNTs contain oxygenated functional groups on their surface, a Lewis acidbase interaction between the CNT surface and aniline is theoretically possible. Pursuing this line of thought further, the low content of oxygenated functional groups on the surface of GMWCNTs implied that the Lewis acidbase interaction, in this case, should be weaker than that observed for MWCNTs. Moreover, all three CNTs exhibited a negative surface charge and thus electrostatically repelled aniline (which was also negatively charged) during the adsorption process. Since GMWCNTs exhibited the highest $\mathrm{pH}_{\mathrm{pzc}}$, they were less affected by this electrostatic repulsion than MWCNTs.

To estimate the effect of solution $\mathrm{pH}$ on the adsorption of aniline and nitrobenzene on CNTs, adsorption experiments were conducted at $\mathrm{pH} 2,3,5,7,9,11$ and 13. The initial solution $\mathrm{pH}$ was adjusted with $1 \mathrm{M} \mathrm{HCl}$ and $1 \mathrm{M}$ $\mathrm{NaOH}$.

As shown in Figure 3, the equilibrium adsorption capacity of CNTs initially increased and then decreased with increasing $\mathrm{pH}$. For aniline, maximal adsorption capacity was determined as $21.3 \mathrm{mg} \mathrm{g}^{-1}$ (SWCNTs; $\mathrm{pH}$ 5); $19.1 \mathrm{mg} \mathrm{g}^{-1}$ (MWCNTs; $\mathrm{pH} 5$ ) and $18.5 \mathrm{mg} \mathrm{g}^{-1}$ (GMWCNTs; pH 7). In the case of nitrobenzene, the equilibrium adsorption capacity slightly decreased with increasing $\mathrm{pH}$, in contrast to the behaviour observed for aniline, since nitrobenzene is a non-ionizable polar organic material that is only weakly electrostatically repelled by CNTs. Therefore, the effect of $\mathrm{pH}$ was insignificant, and the nitrobenzene adsorption capacity was mainly 
determined by the hydrophobic effect and $\pi-\pi$ EDA interactions. For SWCNTs, MWCNTs, and GMWCNTs, the maximal nitrobenzene adsorption capacity was determined as $81.03 \mathrm{mg} \mathrm{g}^{-1}$ (pH 5), $74.28 \mathrm{mg} \mathrm{g}^{-1}$ (pH 5) and $56.83 \mathrm{mg} \mathrm{g}^{-1}(\mathrm{pH} 2)$ respectively.

Notably, the $\mathrm{pH}$ values at which maximal adsorption capacities were observed mainly reflected the $\mathrm{pH}_{\mathrm{pzc}}$ of the three CNTs and the degree of aniline electrolysis, in agreement with the results of previous studies which have shown that at certain $\mathrm{pH}$, organic contaminants containing hydroxyl and amino groups can engage in Lewis acid-base interactions with the surface functional groups of $\mathrm{CNTs}^{43}$. The ionization of functional groups of organic adsorbates prevents them from donating protons to form hydrogen bonds with CNTs. Moreover, when the charges carried by the functional groups of organics are the same as those on the CNT surface, ionization of these functional groups results in enhanced electrostatic repulsion. Therefore, as the initial solution $\mathrm{pH}$ gradually increased, the adsorption of aniline on CNTs became progressively more difficult. Figure 3 demonstrates that the aniline adsorption capacity of SWCNTs and MWCNTs sharply declines with increasing $\mathrm{pH}$ at a value $>5$, which can be ascribed to the progressing ionization of oxygenated functional groups on the CNT surface, which inhibits

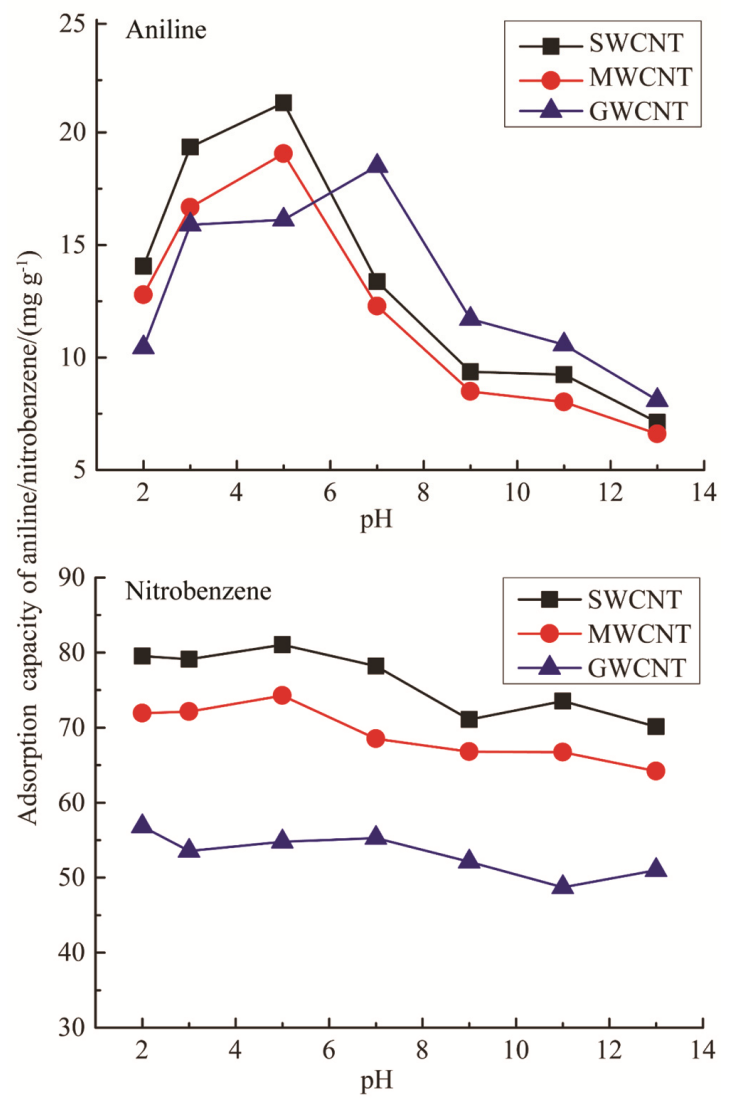

Figure 3. pH-dependent aniline and nitrobenzene adsorption capacity of the studied CNTs. their Lewis acid-base interactions with amino groups. However, the above effect was relatively weakly pronounced for GMWCNTs, which contained an insignificant amount of functional groups, whereas the ionization of surface functional groups enhanced $\pi-\pi$ EDA interactions by increasing surface electron density. Thus, the aniline adsorption capacity of GMWCNTs increases as $\mathrm{pH}$ increases from 2 to 7 . Since the $\mathrm{pH}_{\mathrm{pzc}}$ values of all CNTs were less than 7, it was inferred that the strength of repulsion between aniline and CNTs decreased and that of aniline adsorption on CNTs increased as the $\mathrm{pH}$ approached $\mathrm{pH}_{\mathrm{pzc}}$. Thus, maximum adsorption capacity for all CNTs was reached at $\mathrm{pH}$ close to the corresponding $\mathrm{pH}_{\mathrm{pzc}}$ values.

Herein, we have studied the mechanism of aniline and nitrobenzene adsorption on SWCNTs, MWCNTs and GWCNTs, providing a basis for adsorption-based removal of aromatic organics. The adsorption of aromatic organics on CNTs was shown to be mainly determined by adsorbent-specific surface area, and adsorption behaviour differences between CNTs reflected the ability of their surface hydroxyl and carboxyl groups to enhance the adsorption of certain organic compounds. However, the above organics showed different adsorption behaviours, i.e. the maximum nitrobenzene adsorption capacity (74.2 $\mathrm{mg} \mathrm{g}^{-1}$ ) was much higher than that for aniline $\left(21.5 \mathrm{mg} \mathrm{g}^{-1}\right)$ in view of adsorbate and adsorbent chemical property differences (i.e. amino group presence).

1. Xu, P., Yu, B., Li, F. L., Cai, X. F. and Ma, C. Q., Microbial degradation of sulfur, nitrogen and oxygen heterocycles. Trends Microbiol., 2006, 14, 398-405.

2. Bi, E., Schmidt, T. C. and Haderlein, S. B., Environmental factors influencing sorption of heterocyclic aromatic compounds to soil. Environ. Sci. Technol., 2007, 41, 3172-3178.

3. Rouquerol, J., Rouquerol, F., Llewellyn, P., Maurin, G. and Sing, K. S., Adsorption by Powders and Porous Solids: Principles, Methodology and Applications, Academic Press, 2013.

4. Sillanpaa, M. E., Kurniawan, T. A. and Lo, W. H., Degradation of chelating agents in aqueous solution using advanced oxidation process (AOP). Chemosphere, 2011, 83, 1443-1460.

5. Takht Ravanchi, M., Kaghazchi, T. and Kargari, A., Application of membrane separation processes in petrochemical industry: a review. Desalination, 2009, 235, 199-244.

6. Borukhova, S., Biomass for sustainable applications: pollution remediation and energy. Green Process. Synth., 2014, 3, 305-306.

7. Abhishek, A., Saranya, N., Chandi, P. and Selvaraju, N., Studies on the remediation of chromium (vi) from simulated wastewater using novel biomass of Pinus kesiya cone. Desalin. Water Treat., 2018, 114, 192-204.

8. Saranya, N., Department of Chemical Engineering, N.I.o.T.C., Calicut 673601, Kerala, India, Nakeeran, E., Department of Chemical Engineering, N.I.o.T.C., Calicut 673601, Kerala, India, Giri Nandagopal, M.S., Department of Chemical Engineering, N.I.o.T.C., Calicut 673601, Kerala, India, Selvaraju, N. and Department of Chemical Engineering, N.I.o.T.C., Calicut 673601, Kerala, India, Optimization of adsorption process parameters by response surface methodology for hexavalent chromium removal from aqueous solutions using annona reticulata linn peel microparticles. Water Sci. Technol., 2018, 75, 2094-2107. 
9. Nakkeeran, E., Patra, C., Shahnaz, T., Rangabhashiyam, S. and Selvaraju, N., Continuous biosorption assessment for the removal of hexavalent chromium from aqueous solutions using Strychnos nux-vomica fruit shell. Bioresour. Technol. Rep., 2018, 3, 256260 .

10. Karthik, V., Saravanan, K., Nakkeeran, E. and Selvaraju, N., Biosorption of turquoise blue dye from aqueous solution by dried fungal biomass (Trichoderma harzianum) - kinetic, isotherm and thermodynamic studies. Desalination Water Treatment, 2017, 74, $362-370$.

11. Demirbas, A., Agricultural based activated carbons for the removal of dyes from aqueous solutions: a review. J. Hazard. Mater., 2009, 167, 1-9.

12. Hosseini, S. J., Kokhdan, S. N., Ghaedi, A. M. and Moosavian, S. S., Comparison of multiwalled carbon nanotubes and activated carbon for efficient removal of methyl orange: kinetic and thermodynamic investigation. Fresinius Environ. Bull., 2011, 20, 219 234.

13. Iijima, S., Helical microtubules of graphitic carbon. Nature, 1991, 354, 56-58.

14. Brar, S. K., Verma, M., Tyagi, R. D. and Surampalli, R. Y., Engineered nanoparticles in wastewater and wastewater sludgeevidence and impacts. Waste Manage., 2010, 30, 504-520.

15. Pyrzynska, K., Carbon nanostructures for separation, preconcentration and speciation of metal ions. TrAC-Trends Anal. Chem., 2010, 29, 718-727.

16. Herrero Latorre, C., Alvarez Mendez, J., Barciela Garcia, J., Garcia Martin, S. and Pena Crecente, R. M., Carbon nanotubes as solid-phase extraction sorbents prior to atomic spectrometric determination of metal species: a review. Anal. Chim. Acta, 2012, 749, 16-35.

17. Yu, J. G. et al., Aqueous adsorption and removal of organic contaminants by carbon nanotubes. Sci. Total Environ., 2014, 482483, 241-251.

18. Apul, O. G. and Karanfil, T., Adsorption of synthetic organic contaminants by carbon nanotubes: a critical review. Water Res., $2015, \mathbf{6 8}, 34-55$.

19. Ncibi, M. C. and Sillanpaa, M., Optimized removal of antibiotic drugs from aqueous solutions using single, double and multi-walled carbon nanotubes. J. Hazard Mater., 2015, 298, 102110 .

20. Peng, X., Hu, F., Lam, F. L., Wang, Y., Liu, Z. and Dai, H., Adsorption behaviour and mechanisms of ciprofloxacin from aqueous solution by ordered mesoporous carbon and bamboobased carbon. J. Colloid Interface Sci., 2015, 460, 349-360.

21. Upadhyayula, V. K., Deng, S., Mitchell, M. C. and Smith, G. B., Application of carbon nanotube technology for removal of contaminants in drinking water: a review. Sci. Total Environ., 2009, 408, 1-13.

22. Balarak, D., Mostafapour, F., Bazrafshan, E. and Saleh, T. A., Studies on the adsorption of amoxicillin on multi-wall carbon nanotubes. Water Sci. Technol., 2017, 75, 1599-1606.

23. Agarwal, S., Tyagi, I., Gupta, V. K., Dehghani, M. H., Jaafari, J., Balarak, D. and Asif, M., Rapid removal of noxious nickel (II) using novel $\gamma$-alumina nanoparticles and multiwalled carbon nanotubes: kinetic and isotherm studies. J. Mol. Liquids, 2016, 224, 618-623.

24. Balarak, D., Mahdavi, Y., Bazrafshan, E., Mahvi, A. H. and Esfandyari, Y., Adsorption of fluoride from aqueous solutions by carbon nanotubes: determination of equilibrium, kinetic, and thermodynamic parameters. Adsorption, 2016.

25. Ajayan, P., Nanotubes from carbon. Chem. Rev., 1999, 99, 17871800 .

26. Bahgat, M., Farghali, A. A., El Rouby, W. M. A. and Khedr, M. H., Synthesis and modification of multi-walled carbon nano-tubes (mwcnts) for water treatment applications. J. Anal. Appl. Pyrol., 2011, 92, 307-313.
27. Ma, X. M., Tsige, M., Uddin, S. and Talapatra, S., Application of carbon nanotubes for removing organic contaminants from water. Mater. Express, 2011, 1, 183-200.

28. Ruelle, B., Peeterbroeck, S., Godfroid, T., Bittencourt, C., Hecq, M., Snyders, R. and Dubois, P., Selective grafting of primary amines onto carbon nanotubes via free-radical treatment in microwave plasma post-discharge. Polymers-Basel, 2012, 4, 296-315.

29. Dong, L. Z. C., Discussion on the difference between single wall carbon nanotubes and multi walled carbon nanotubes. Friend Sci. Amateurs, 2012, 14-15.

30. Terrones, M., Science and technology of the twenty-first century: synthesis, properties and applications of carbon nanotubes. Annu. Rev. Mater. Res., 2003, 33, 419-501.

31. Zhao, J., Preparation and application of high quality multi walled carbon nanotubes, Shanghai Jiao Tong University, China.

32. Bittner, E. W., Smith, M. R. and Bockrath, B. C., Characterization of the surfaces of single-walled carbon nanotubes using alcohols and hydrocarbons: a pulse adsorption technique. Carbon, 2003, 41, 1231-1239.

33. Peng, X. J. J. and Luan, Z. K., Application of carbon nanotubes in the field of water treatment materials. Prog. Chem., 2009, 19871992.

34. Yang, K. and Xing, B., Adsorption of organic compounds by carbon nanomaterials in aqueous phase: polanyi theory and its application. Chem. Rev., 2010, 110, 5989-6008.

35. Zhang, L. L. X. and Feng, J. W., The influence of graphitization on the structure and electrical properties of carbon nanotubes. J. Inorg. Mater., 2009, 535-538.

36. Barrett, E. P., Joyner, L. G. and Halenda, P. P., The determination of pore volume and area distributions in porous substances. I. Computations from nitrogen isotherms. J. Am. Chem. Soc., 1951, 73, 373-380.

37. Azarpira, H., Mahdavi, Y., Khaleghi, O. and Balarak, D., Thermodynamic studies on the removal of metronidazole antibiotic by multi-walled carbon nanotubes. Pharm. Lett., 2016, 8, 107-113.

38. Balarak, D., Bazrafshan, E., Mahdavi, Y., Lalhmunsiama and Lee, S. M., Kinetic, isotherms and thermodynamic studies in the removal of 2-chlorophenol from aqueous solution using modified rice straw. Desalin. Water Treat., 2017, 63, 203-211.

39. Mohammadi, L., Bazrafshan, E., Noroozifar, M., AnsariMoghaddam, A., Barahuie, F. and Balarak, D., Adsorptive removal of benzene and toluene from aqueous environments by cupric oxide nanoparticles: kinetics and isotherm studies. J. Chem., 2017, 2017.

40. Padwa, A. and Pearson, W., The chemistry of heterocyclic compounds, synthetic applications of 1,3-dipolar cycloaddition chemistry toward heterocycles and natural products. John Wiley.

41. Chen, W., Duan, L. and Zhu, D., Adsorption of polar and nonpolar organic chemicals to carbon nanotubes. Environ. Sci. Technol., 2007, 41, 8295-8300.

42. Wu, W., Chen, W., Lin, D. and Yang, K., Influence of surface oxidation of multiwalled carbon nanotubes on the adsorption affinity and capacity of polar and nonpolar organic compounds in aqueous phase. Environ. Sci. Technol., 2012, 46, 5446-5454.

43. Chen, W., Duan, L., Wang, L. and Zhu, D., Adsorption of hydroxyland amino-substituted aromatics to carbon nanotubes. Environ. Sci. Technol., 2008, 42, 6862-6868.

ACKNOWLEDGEMENTS. We thank the National Special Project for Science and Technology on Water Pollution Control and Management (2017ZX07102-003), China for financial support.

Received 12 July 2018; revised accepted 22 May 2019

doi: $10.18520 / \mathrm{cs} / \mathrm{v} 117 / \mathrm{i} 4 / 683-689$ 